\title{
EDITORIAL
}

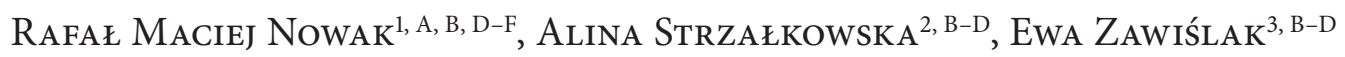

\section{Treatment Options and Limitations in Transverse Maxillary Deficiency}

\section{Możliwości i ograniczenia leczenia niedorozwoju poprzecznego szczęk}

${ }^{1}$ Department of Maxillofacial Surgery, Wroclaw Medical University, Wrocław, Poland

${ }^{2}$ Private Practice "Orthosmile", Wrocław, Poland

${ }^{3}$ Department of Maxillofacial Surgery, Wroclaw Medical University, Wrocław, Poland

A - research concept and design; $\mathbf{B}$ - collection and/or assembly of data; $\mathbf{C}$ - data analysis and interpretation;

$\mathbf{D}$ - writing the article; $\mathbf{E}$ - critical revision of the article; $\mathbf{F}$ - final approval of article

\begin{abstract}
Maxillary constriction is clinically manifested as a narrow palate and a high palatal vault, a complete unilateral or bilateral crossbite, dark buccal corridors, unaesthetic appearance and a significant disproportion between the width of the upper and lower dental arches. Structural maxillary abnormality may also be associated with such functional impairments as food chewing disorder, nose breathing problems and a development of a mouth breathing habit. The purpose of this paper is to present and discuss the most common therapeutic approaches in the management of transverse maxillary deficiency.

The increased transverse dimension may be achieved orthodontically by expansion, with surgical assistance - surgically assisted rapid maxillary expansion - or using purely surgical techniques. Surgical options include transpalatal distraction and segmented maxillary osteotomy. Orthodontic maxillary expansion is effective at developmental age due to the continuous bone growth, until the closure of the midpalatal suture. As the risk of orthodontic treatment failure is higher in adults with maxillary constriction, these patients make good candidates for surgery. The optimum maxillary expansion approach should be chosen based on such unambiguous criteria as patient's age, severity of deformity and the skills of the therapeutic team (Dent. Med. Probl. 2015, 52, 4, 389-400).
\end{abstract}

Key words: rapid maxillary expansion, maxillary constriction, transpalatal distraction, surgically assisted rapid maxillary expansion.

Słowa kluczowe: zwężenie szczęki, poszerzenie szczęki, dystrakcja przezpodniebienna, chirurgicznie wspomagane poszerzenie szczęki.

Transverse maxillary deficiency can occur as an isolated anomaly of the upper dental arch or as a component of a complex skeletal malformation. The causes of the condition include abnormal habits, dysfunctions and parafunctions (such as thumb sucking and mouth breathing habits), muscle disorders and congenital syndromes (Apert Syndrome, Cruzone Syndrome, Marfan Syndrome, etc.). Although it is a known complication in patients after treatment of the primary or secondary cleft palate, it may also present as a comorbitity to the cleft palate. Regardless of the cause, the transverse maxillary deficiency is typically manifested as the complete unilateral or bilateral crossbite, a narrow palate and a high palatal vault, dark buccal corridors and a significant disproportion between the width of the upper and lower dental arches (Fig. 1.).

Maxillary constriction with the resulting lowered tongue posture may contribute to the development of an abnormal breathing pattern - persistent mouth breathing and the hypotension of the orbicularis oris muscle. Conversely, the lowered tongue position secondarily affects the shape of the upper dental arch leading to crowding of teeth, especially in the anterior segment. If left un- 


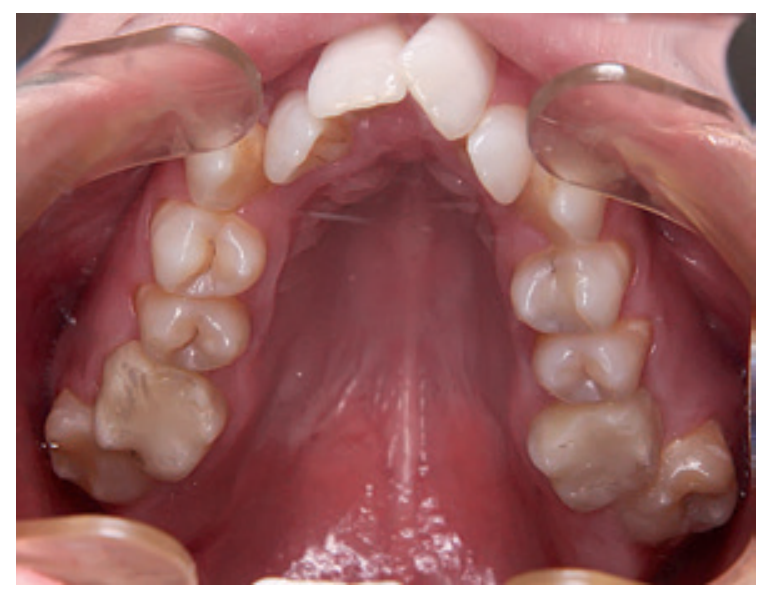

treated, maxillary constriction may not only lead to malocclusion and aesthetic defects. More serious sequelae involve ENT disorders, nose breathing problems and sleep apnoea syndrome. The estimated prevalence of maxillary constriction in Europe ranges between 13\% and 23\% [1].

Therapeutic strategies used in the management of transverse maxillary deficiency belong to three different approaches: orthodontic methods (SOE - slow orthodontic expansion or SME - slow maxillary expansion, RPE - rapid palatal expansion or RME - rapid maxillary expansion), surgically assisted orthodontic methods (SARPE - surgically assisted rapid palatal expansion or SARME - surgically assisted rapid maxillary expansion) and surgical methods (TPD - transpalatal distraction, LFI - E-segmented Le Fort I osteotomy with expansion) [2]. The choice of the appropriate method should be based on several factors, including patient's age, severity of deficiency, the expertise of the therapeutic team as well as patient's expectations and attitude.

The published data does not determine a conclusive age limit, when the orthodontic maxillary expansion is effective and safe. Clinicians experienced in treating maxillary constriction suggest 14-18 years of age as the upper age limit for orthodontic management. At a more advanced age, in adults treated with rapid palatal expansion (RPE), the risk of dento-periodontal complications and the recurrence of anomaly increases significantly [3-8]. The complications may include alveolar process atrophy, especially on the buccal side, root resorption, gingival recession, buccal inclination and extrusion of teeth affected by the pressure force of the appliance screw. In order to avoid treatment failure in adults, surgically assisted treatments were introduced, as well as bone-anchored, force-generating devices which do not adversely affect lateral teeth and periodontium.

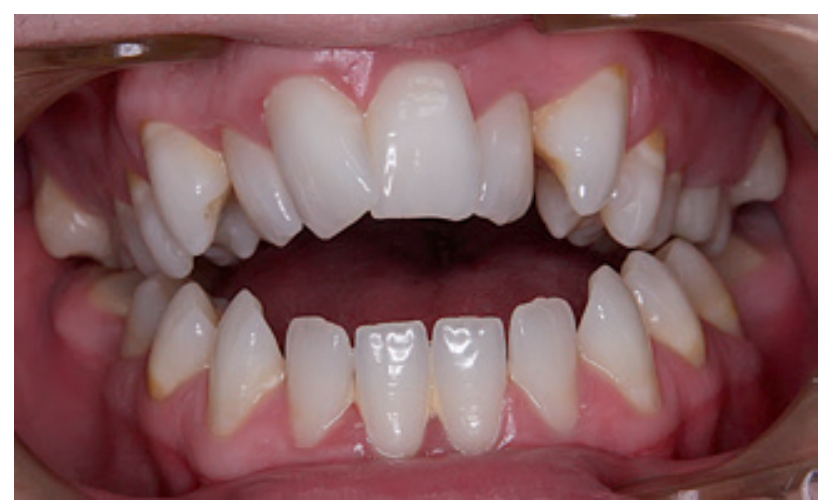

Fig. 1. A typical example of a significant maxillary constriction

\section{Maxillary Expansion Methods}

\section{Orthodontic Approaches}

Orthodontic treatment alternatives are intended for the patients at developmental age of an intensive bone growth. The most commonly used orthodontic maxillary expansion methods include slow orthodontic (maxillary) expansion (SOE, SME) and rapid palatal (maxillary) expansion (RPE, RME) [9].

The orthodontic maxillary expansion utilises different appliances (expanders) as a source of pressure force. There are three main types of bonded appliances used for maxillary expansion: baseplate appliance with a midline expansion screw, thick-wire appliances such as W-arch appliance (Feder), bi-helix expander, NiTi expander and Hyrax screw expanders (Hass or Biederman appliance). They work on a roughly similar principle. The pressure force originating from the expansion screw and/or springs is transduced onto the premolars and molars, and eventually - the mid-palatal suture. The expansion screw appliances can be used both for the rapid palatal expansion (RPE) and slow orthodontic expansion (SOE). However, the thick-wire appliances are intended for free expansion only and their various modifications are very common nowadays $[5,10,11]$.

There are tooth-borne, tooth-tissue-borne and bone-borne maxillary expanders. Two first groups are jointly referred to as tooth-anchored maxillary expansion (TAME). The third one belongs to the category of bone-anchored maxillary expansion (BAME). The first group involves rigid appliances whose force is generated by the screw (Hyrax appliance) and flexible appliances whose force is generated by the thick arch wires (Bi-helix, Quad-helix). The tooth-tissue-borne expanders are made of acrylic. The force is generated by the screw, and the anchor, which can be either lim- 


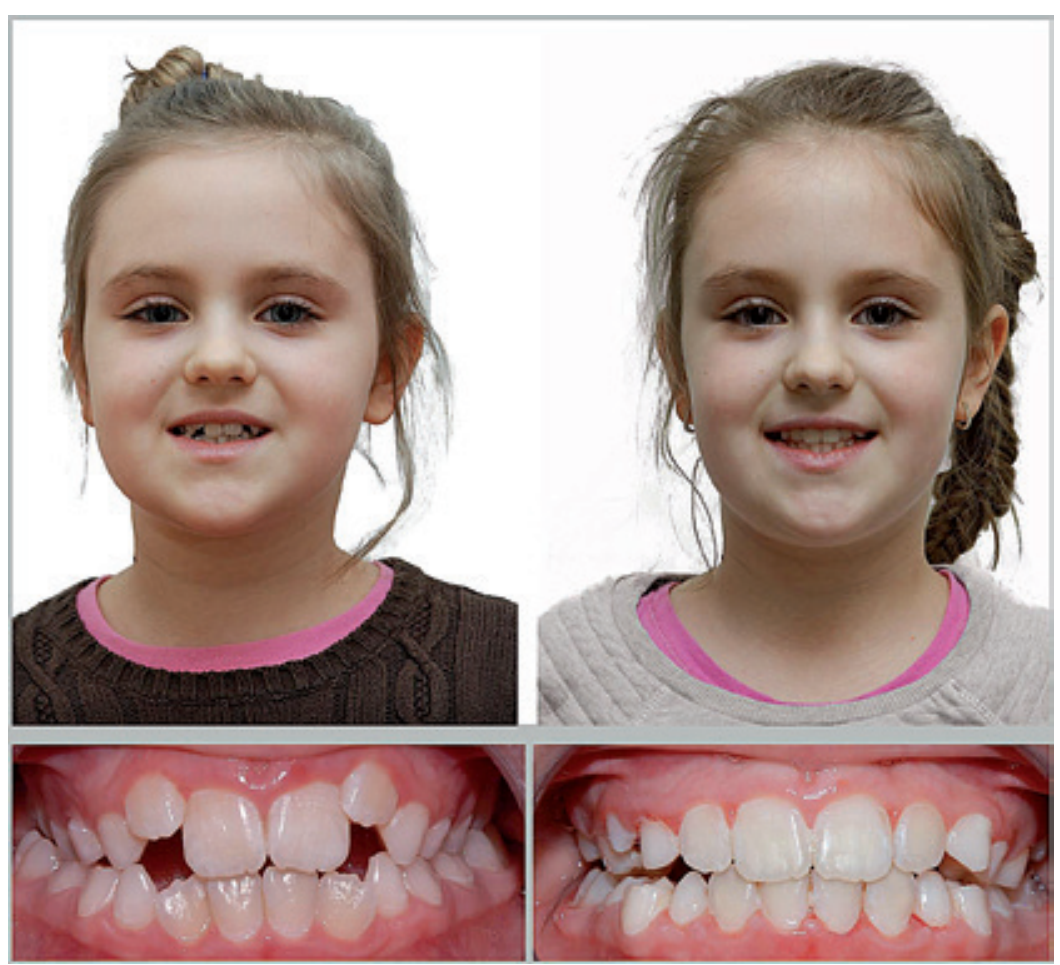

Fig. 2. 9-year old girl with maxillary constriction treated with tooth-tissue borne Hyrax expander (Hamilton plate)
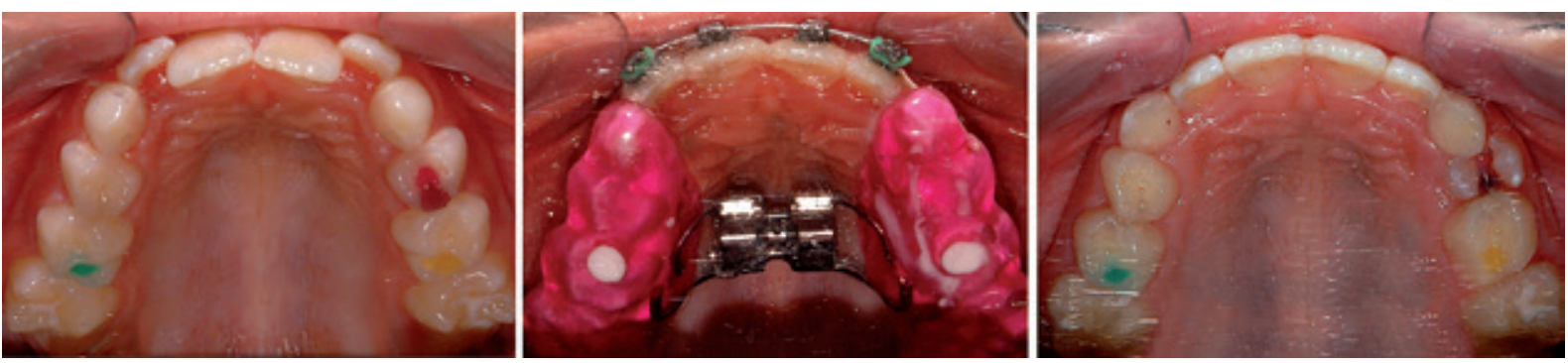

ited to tooth-bonded bands (Hamilton plate) or stretch from these bands onto the palate (Haas appliance), is made of acrylic [12]. The third group of bone-borne expanders includes all screw-based appliances, which are anchored to the palatal bone using orthodontic microscrews. The resistance of perimaxillary sutures limits the orthopaedic effect of the latter on the maxillary bone. The studies do not show differences between the toot-borne and tooth-tissue-borne appliances [13, 14].

A typical Hyrax appliance has a screw along the mid-palatal suture with two $1.5 \mathrm{~mm}$ thick wires stretching out of it, which are bonded to the abutment teeth (usually first permanent molars and first premolars in individuals with mixed teeth or complete permanent teeth) with the rings. The rings on the abutment teeth are joined with the $0.7-0.9 \mathrm{~mm}$ thick wire welded onto them. Another alternative is Hyrax appliance with acrylic splints (used e.g. in individuals with primary teeth only or with early mixed teeth), which does not require rings and aims at reducing lateral teeth inclination [12]. After Hyrax appliance is fixed onto the teeth, the patient or his parents/carers are instructed how to activate it.
The rapid palatal expansion (RPE) involves screw activation by 0.5 to $1 \mathrm{~mm}$ on a daily basis. The most common expansion protocol involves expanding the screw by a quarter-turn twice daily, which translates into an expansion of $0.4 \mathrm{~mm}$ a day. Active treatment is continued until the desired arch widths and interarch relationships are achieved (approx. 2-3 weeks). A transverse force vector whose value ranges between 4.5 and $9 \mathrm{~kg}$ is applied onto the mid-palatal suture causing it to separate. A clinical manifestation of a rapid maxillary expansion is the occurrence of diastema. An active treatment phase is followed by the retention period of two-to-four months. During that period, the full-thickness bone tissue is formed between the separated palatal shelves.

Both Hyrax screw and Haas appliances induce wedge-shaped palatal expansion with anterior opening, where $50 \%$ of expansion affects the anterior and $36 \%$ - the posterior area [14]. It is a paradigm that the process can take place only in patients with incomplete palatal suture ossification. It is thought that the palatal suture is completely closed at the age of 15 and 16, in girls and boys, respectively [15]. However, there are also reports 


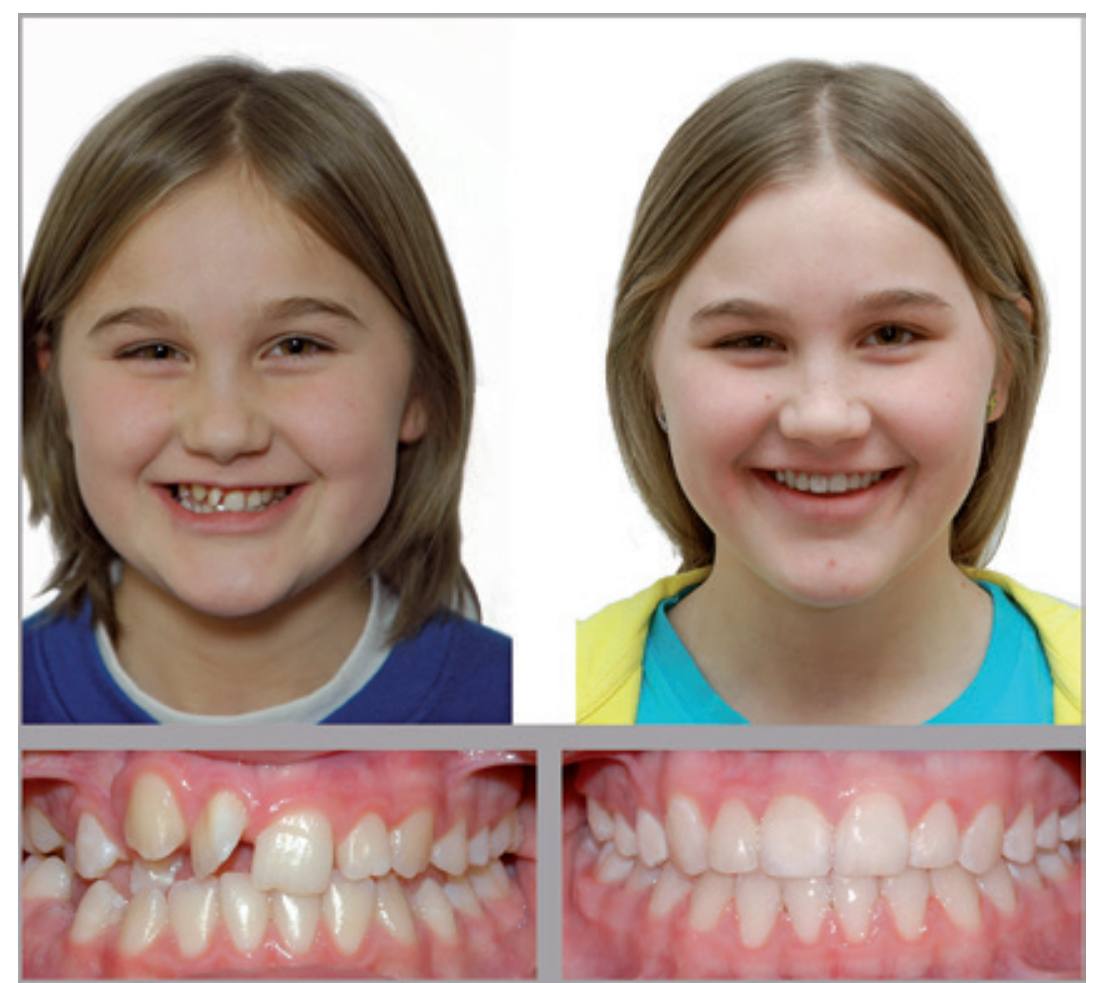

Fig. 3. 11-year old girl with maxillary constriction and teeth transposition treated with tooth borne Hyrax expander
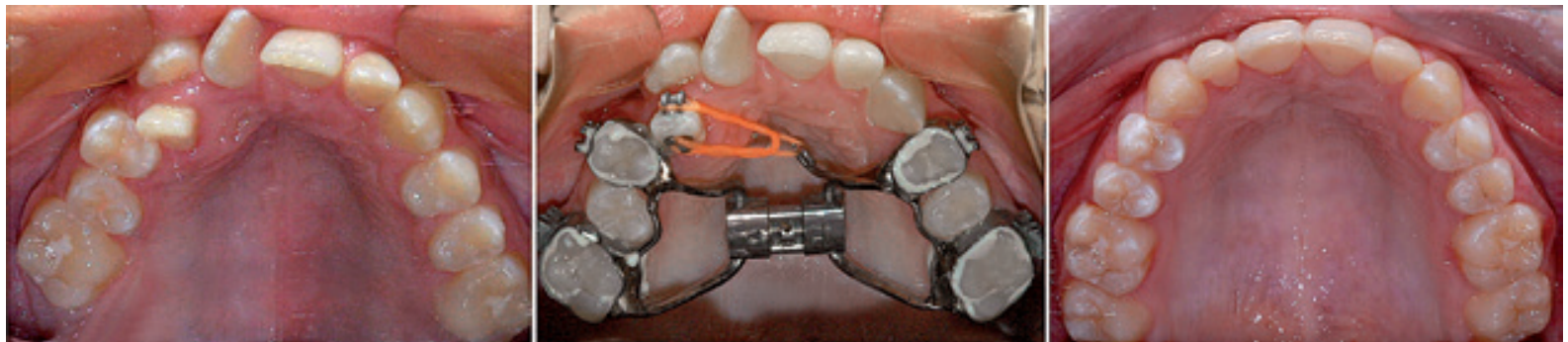

indicating that palatal suture ossification is complete by 13 years of age [14].

Transverse maxillary expansion combined with maxillary protraction is one of the treatment approaches in skeletal class III malocclusion.

This type of deformity should be corrected as early as possible [16]; therefore, Hyrax appliances should be used for palatal expansion in patients with mixed teeth. The treatment aims at expanding the midface, correction of a concave facial profile and crossbite (in lateral segments), as well as improving the overjet [17]. Unilateral crossbite may cause a functional shift, which becomes a habitual rest position. This impairs proper chewing function and induces temporomandibular joint problems. Early intervention enables proper positioning of mandibular condyles within the mandibular fossa, which will contribute to the normal growth and development [18].

When used in patients after the growth and developmental phase, RME may lead to numerous complications. These include anchor tooth mobility, inclination and extrusion, thinning of the cortical plate in the alveolar processes, periodon- tal defects manifesting as gingival recession, dehiscence or clinical attachment loss, pulp vitality loss, palatal necrosis, pain and unstable treatment outcomes. The patients with gingivitis and inadequate oral hygiene are particularly susceptible to pathological mobility of the appliance-bearing teeth $[15,19,20]$.

That is why RME is considered an effective orthopaedic treatment only in growing patients with maxillary transverse deficiency. The main purpose of this expansion technique is to open the palatal suture which enables the formation of a normal, stable maxillary width. Furthermore, the rapid maxillary expansion has a proven beneficial effect on the volume and shape of the upper respiratory tract. The maxilla forms approx. half of skeletal structure of nasal cavity. As the palatal suture opens, the lateral walls of the nasal cavity are automatically pushed outwards which increases the nasal cavity volume. As a result, there is less resistance to the airflow in this segment of the respiratory tract. The improved breathing pattern positively affects the craniocervical junction, which has an abnormally large angular measurement in patients with respiratory dys- 


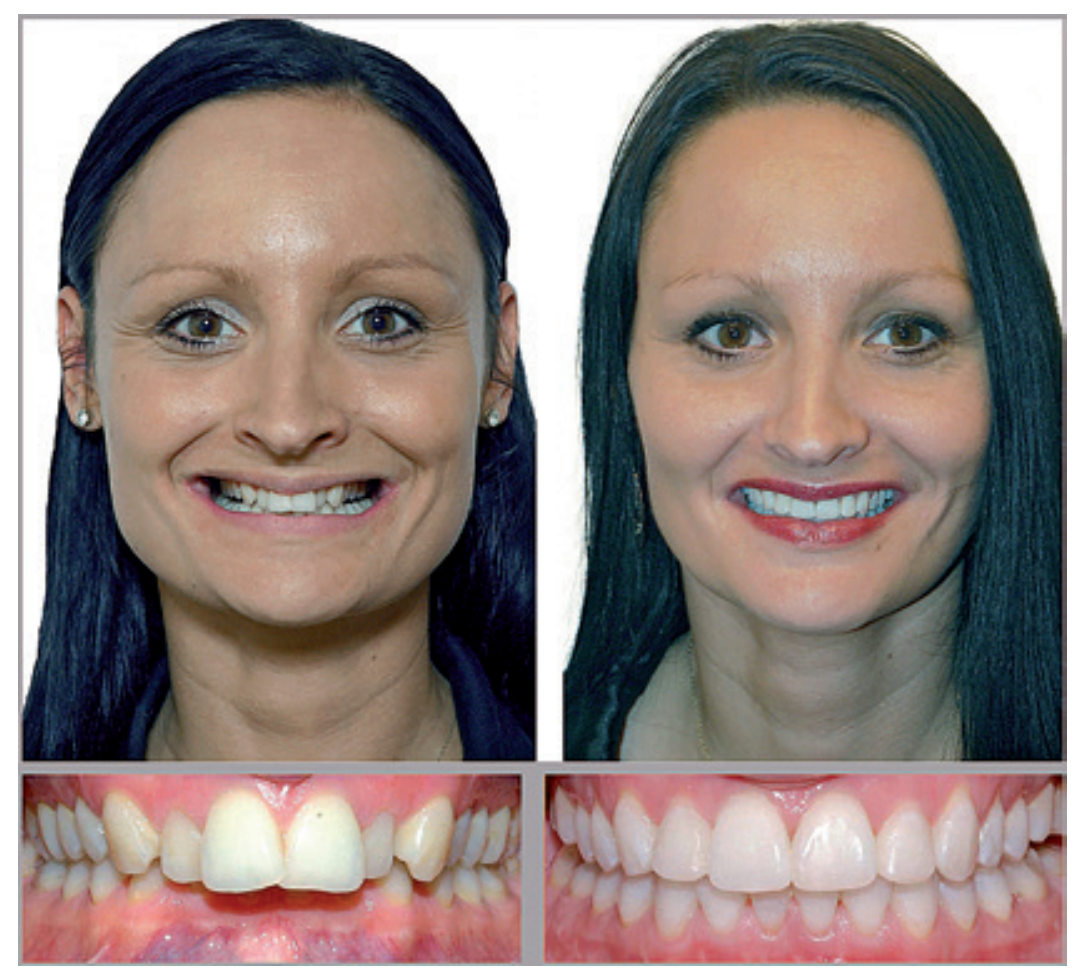

Fig. 4. 35-year old woman with class II division 1 malocclusion with maxillary constriction treated with tooth borne Hyrax expander - RME method
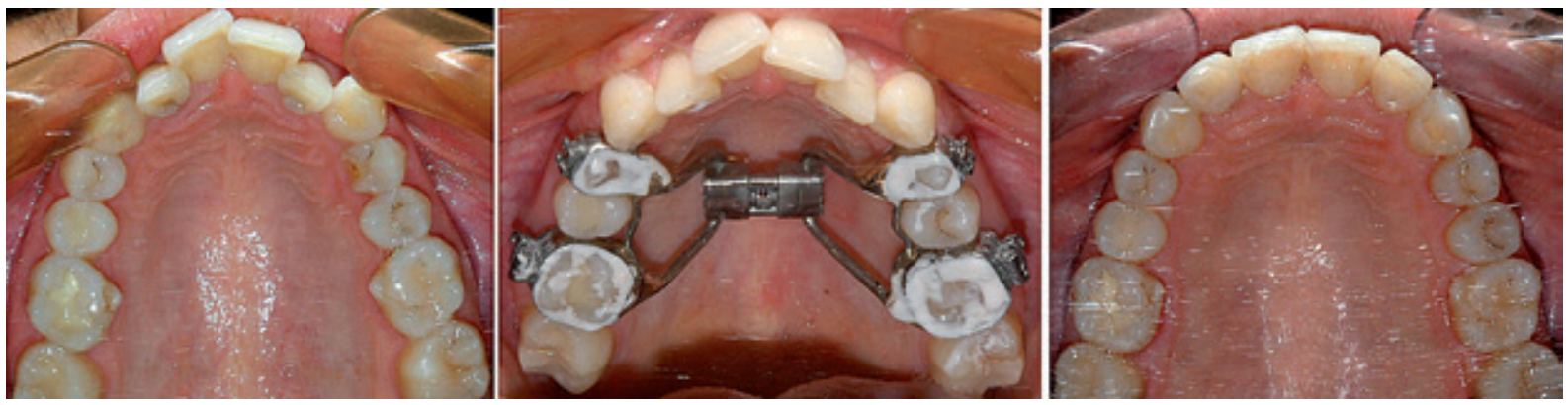

function. Further research is needed in order to confirm the stability of the observed treatment-induced changes [21] (Fig. 2-4).

Another orthodontic approach using hyrax appliance is slow orthodontic (maxillary) expansion (SOE, SME). Treatment protocol in slow orthodontic expansion involves screw activation up to $1 \mathrm{~mm}$ each week, which generates a force of 900 to $200 \mathrm{~g}$ applied onto the midpalatal suture. The stage of active treatment is appropriately longer whereas the retention period lasts for 3 months. The majority of achieved expansion can be attributed to tooth and alveolar process tilt. The orthodontic to orthopaedic expansion ratio ranges from 6:1 to 10:1 [22-24]. Other authors report it to be 2:1 [25]. Therefore, the published data shows a significant discrepancy.

Recurrence, due to the palatal soft tissue flexibility and dentoalveolar inclination, may constitute $30-40 \%$ of the achieved expansion, so the therapy should aim at overcorrection (Fig. 5).

In 1975 Rickets [26] presented maxillary expander resembling contemporary quadhelix. It was an alternative to hyrax appliance in cases requiring maxillary expansion and gave better results in individuals with early mixed teeth. It generated less force but of more physiological nature, which helped achieve more stable correction.

Modern quadhelix is a thick-wire appliance manufactured of $0.9 \mathrm{~mm}$ thick stainless steel wire. It consists of four ring loops and two arms supported on or close to the lingual surfaces of upper premolars. The entire structure is placed within the inner tubes, on the rings of the first maxillary premolars. Quadhelix appliances come in 5 sizes ( 1 do 5), whereas sizes 2 and 3 are the most commonly used [27]. They do not require additional procedures (e.g. taking dental impressions). Instead, they can be fixed and fully activated during a single appointment.

Quadhelix generates milder force than hyrax expander and the achieved expansion ranges between 0.4 and $1 \mathrm{~mm}$. These appliances have more orthodontic (i.e. dentoalveolar) effect, the expansion progresses more slowly with the effect being visible in teeth posture. 


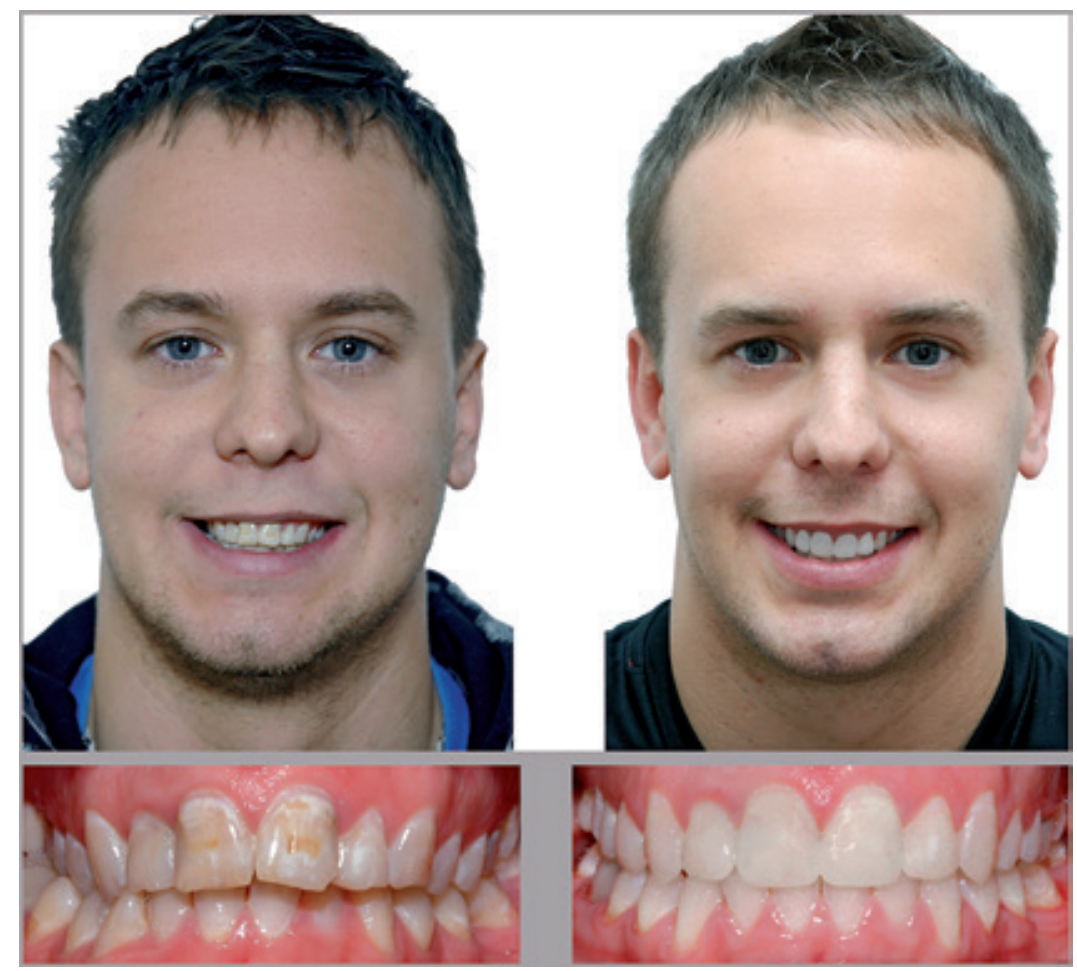

Fig. 5. 27-year old man with crossbite treated with tooth borne Hyrax expander - SME method
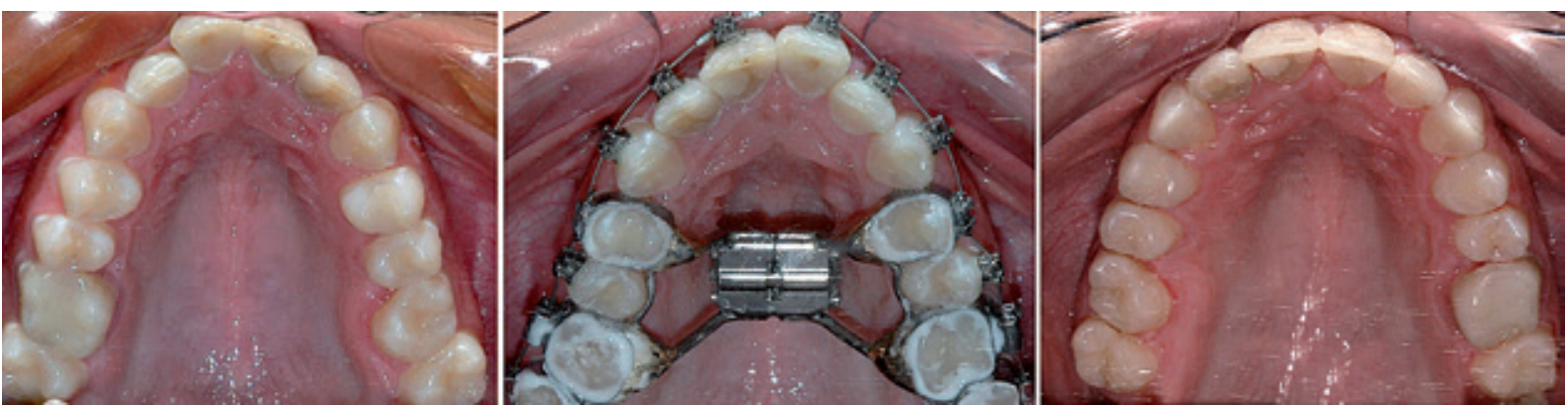

Bihelix expander is a simplified version of quadhelix, without the teardrop-shaped loops bonded to molars, which makes it easier to adjust the appliance in patient's mouth and offers improved comfort. According to Huyhn et al., [18] using bihelix appliance to for maxillary expansion by $5 \mathrm{~mm}$ is associated with the $25 \%$ recurrence at 2 years (Fig. 6).

The non-surgical maxillary expansion in adults is widely debated in the literature. It is commonly thought that the palatal suture distraction is impossible once the growth and development period has finished. Surgical maxillary expansion is considered a treatment of choice in adults with jaw discrepancy [29].

The non-surgical maxillary expansion in adults follows a different course to the one observed in patients at the active growth stage. In adult patients, the observed maxillary expansion is primarily associated with the buccal tilt of the alveolar processes and the resulting identical tooth tilt in the lateral arch segments. Alveolar process tilt starts approximately at the upper third of the palatal vault measuring from the palatal suture.
Biologically, orthopaedic maxillary expansion in adults is based on the occurrence of resorption areas on the palatal surface of the alveolar bone and aposition zones on its buccal surface.

It was shown that in children, $50 \%$ of expansion is manifested in the palatal suture, whereas the remaining $50 \%$ is manifested within the alveolar processes. This challenges the commonly held orthodontic conviction that maxillary expansion primarily involves the palatal suture [29].

Independent studies by Iseri \& Özsoy and Krebs [30], who used metal implants in juvenile patients at the mean age of 14.5 years, showed that only $40 \%$ to $50 \%$ of expansion resulting from $\mathrm{RME}$ is of skeletal nature. They determined that the remaining expansion is manifested within the alveolar processes. The study by Handelman [29] on adult patients after surgically unassisted RME showed that the entire expansion was manifested within the alveolar processes.

Kartalian et al. [31] showed $2 \mathrm{~mm}$ of palatal suture expansion per every $5 \mathrm{~mm}$ of upper arch expansion in growing patients. Such conclusions 


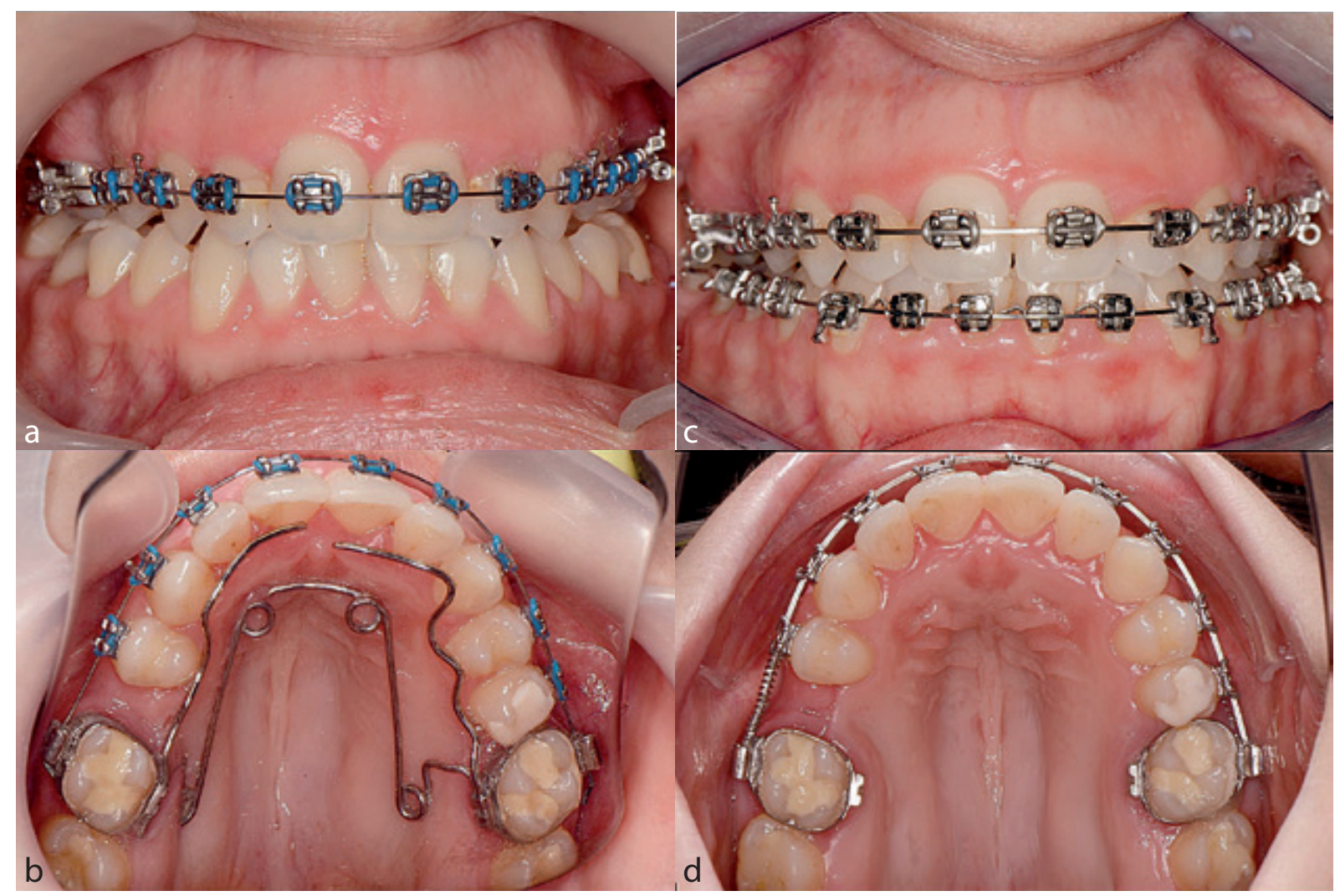

Fig. 6. 20-year old woman with bilateral crossbite treated with quadhelix appliance (courtesy of dr Liwia Minch, orthodontist)

support the use of RME in selected cases even after the end of growth period and may serve as a trigger to change attitudes and mindset.

\section{Surgically Assisted Approaches}

The general assumption of the surgically assisted approaches involves the elimination of bone resistance within the maxilla before orthodontic treatment is commenced. For SARPE/SARME therapies, teeth-anchored hyrax appliances are used. Maxillary and midpalatal suture expansion is achieved after previous surgical mobilisation and elimination of bone resistance by dissection followed by the subsequent gradual screw activation. In line with craniofacial architecture and biomechanics, the strongest midfacial bone resistance areas are located within the so-called Sicher pillars - canine pillar, zygomatic pillar and pterygoid pillar as well as the midpalatal suture.

The first stage of treatment involves a design and fixation of hyrax appliance on the patient's teeth. Afterwards, the patient is referred for the surgery.

The surgery is performed under general anaesthesia, after prior injecting a mixture of local anaesthetic and vasoconstrictive agents into the oral vestibular and palatal soft tissues. As a part of preferred strategy for perioperative infection prevention, an antibiotic is administered intravenously 30 minutes before surgical tissue dissection. The surgery is typically performed through either buccal sulcus approach or the upper labial frenulum approach. After the incision to the mucosal membrane, the inferior margin of the piriform aperture, as well as the anterior and lateral maxillary surfaces, are exposed (buccal sulcus approach). Next, the maxillary bone resistance is eliminated by cutting along the line that goes bilaterally through the piriform aperture (lateral nasal wall), anterior wall of the maxillary sinus, zygomaticoalveolar crest and the lateral surface of the maxilla (that is, along the LeFort I osteotomy line). After the incision to the mucosal membrane of the upper labial frenulum, the midpalatal suture is surgically separated and the mobility of both maxillas is controlled. During the surgery, the appliance is activated until the diastema of approx. $1 \mathrm{~mm}$ is achieved. Finally, the wound is sutured with 4.0 absorbable sutures. After the postoperative latency of 6-8 days, active treatment is commenced. The hyrax screw activation protocol involves expanding the screw by quarter-turn twice daily, which translates into an expansion of $0.4 \mathrm{~mm}$ a day. After the intended anterior and posterior width dimensions are achieved, the appliance remains in place 


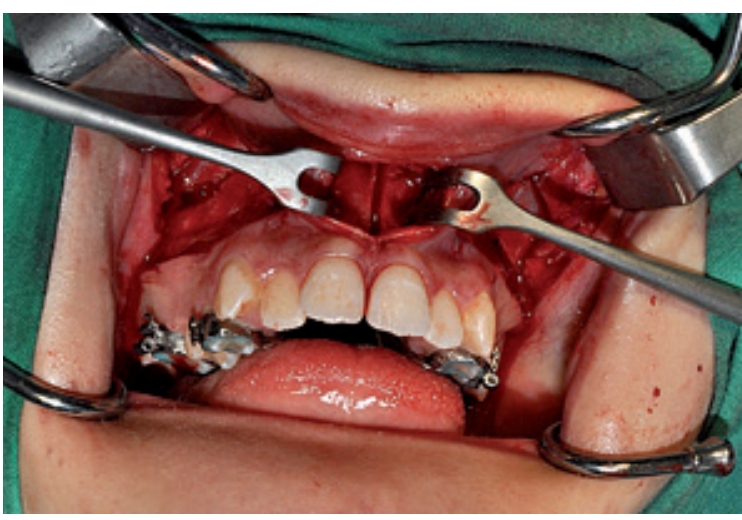

Fig. 7. The midpalatal suture osteotomy line for SARPE/SARME
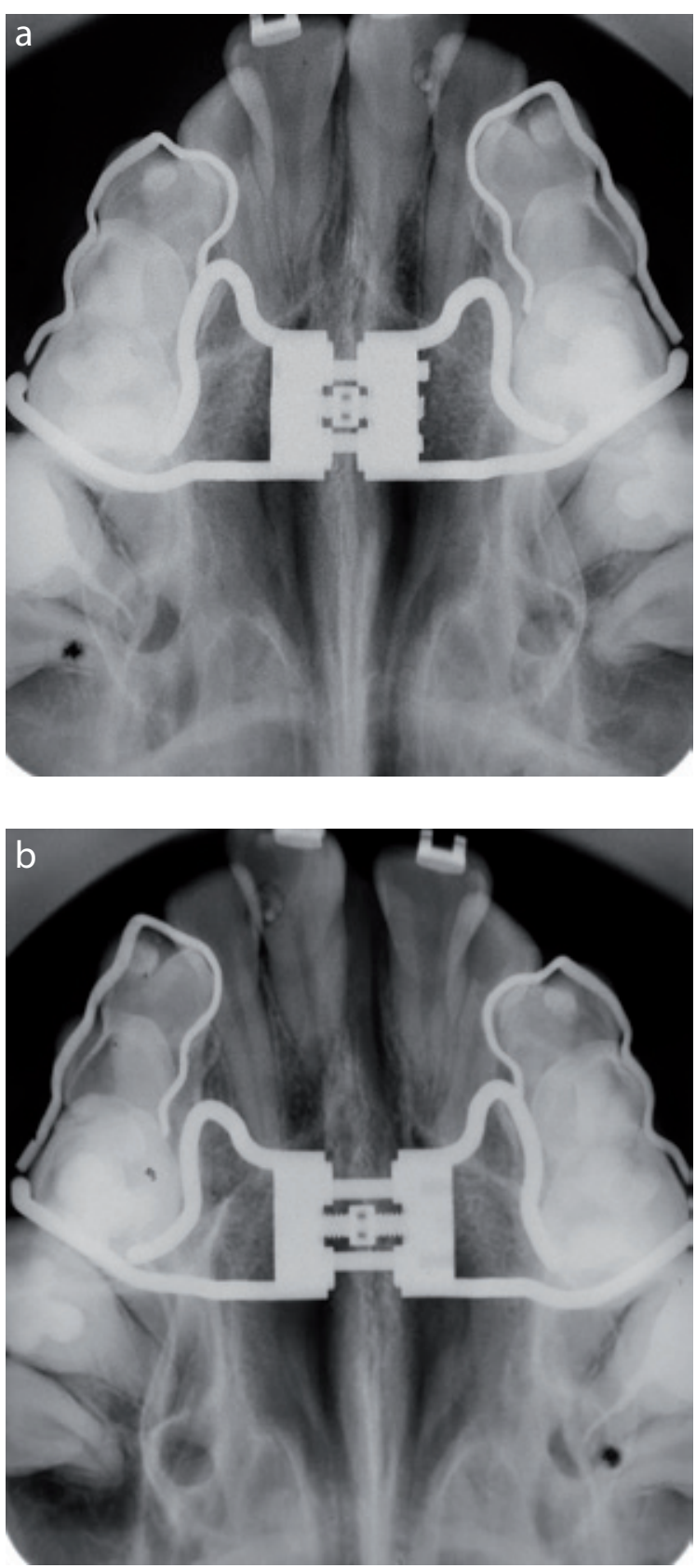

as a part of retention for at least 6 weeks. Further orthodontic treatment may be commenced after the retention period (Fig. 7-8).

\section{Surgical Approaches}

Surgical maxillary expansion is actually limited to two surgical techniques of segmented maxillary osteotomy and transpalatal distraction (TPD), and their modifications. The first of these methods enables increasing the maxillary transverse dimension and correction of maxillary posture in other planes at the same time. The surgery is performed after previous planning a new maxillary posture and a vertical plane's point of intersection on the plaster models.

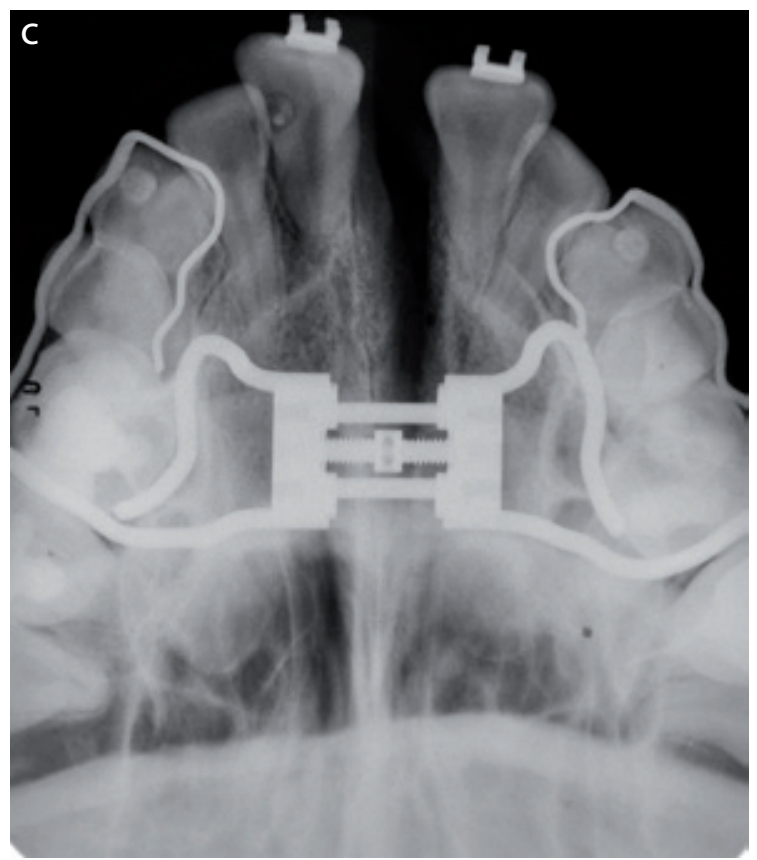

Fig. 8. Palatal x-ray: a) before SARPE, b) on day 7 following SARPE, c) on day 14 following SARPE 

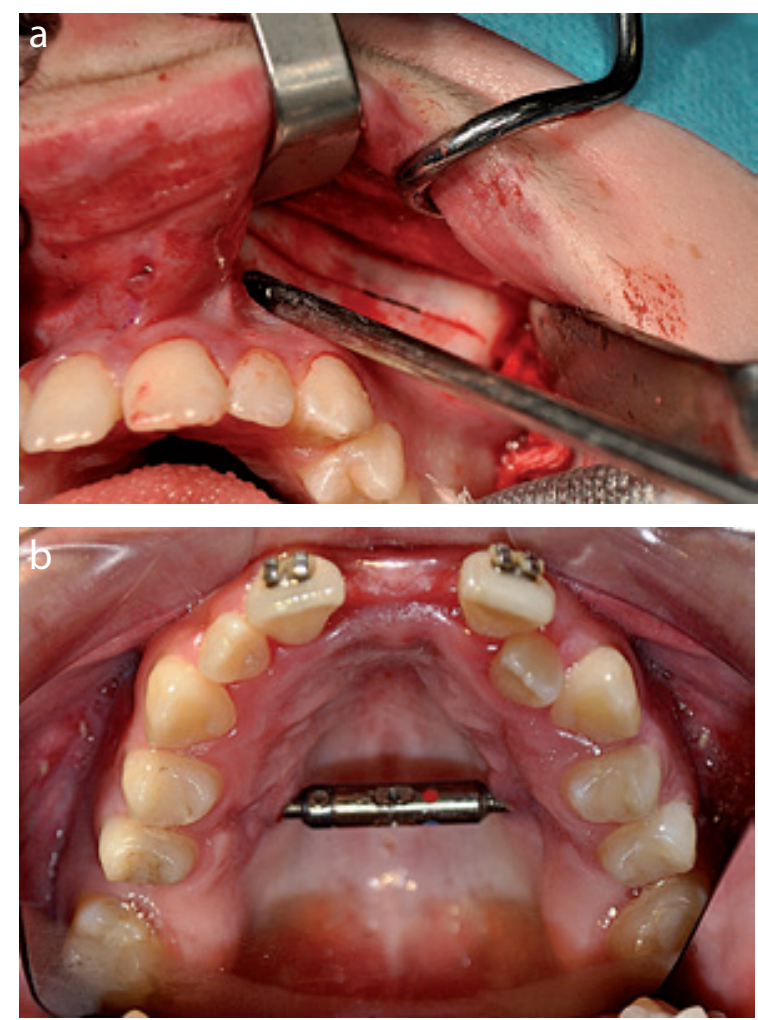

Fig. 9. Maxillary expansion using a transplalatal distractor: a) LF I osteotomy line, b) a distractor fixed onto the palate

The surgical procedure involves performing a total maxillary osteotomy (Le Fort I) with down fracture, dissecting the hard palate and its fusion after new anatomical relationships have been established as planned. Segmented maxillary osteotomy is usually performed as a stage of a larger procedure aimed at correcting a complex skeletal deformity and is typically combined with some mandibular corrective surgery. The limitations of this method include: the insignificant expansion hindered by the palatal mucous membrane, which is not susceptible to stretching; a relatively complex surgical procedure, and the risk of recurrence unless there is a stable postoperative occlusion.

The second method is the transpalatal distraction (TPD) introduced by M. Mommaerts [2] in 1999 as a treatment of severe maxillary constriction in adults. The surgery is performed under general anaesthesia and the procedure itself involves all the stages mentioned previously for surgically assisted rapid palatal expansion (SARME) - a subtotal maxillary osteotomy (Le Fort I) and a separation of the midpalatal suture. However, unlike in SARME, the bone-anchored transpalatal distractor, a force-generating appliance is fixed perpendicularly to the midpalatal suture line for this procedure. The surgical treatment ends with the fixation of the distractor to the palate and its subsequent activation until approx. $1.0 \mathrm{~mm}$ wide
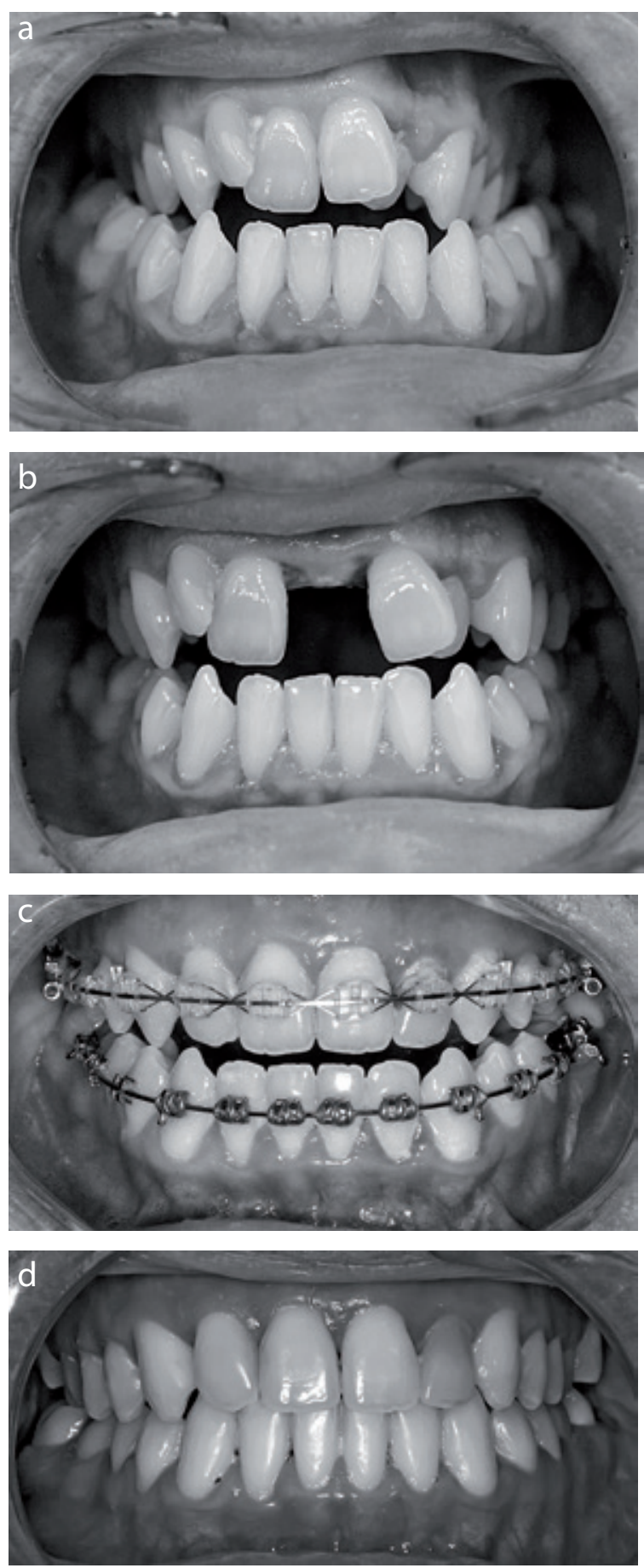

Fig. 10. Complete treatment with tpd and bimaxillary surgery: a) bite before treatment, III class malocclusion, severe crowding and maxillary constriction; b) bite after transpalatal distraction; c) after a complete orthodontic treatment, as a part of preparation for orthognatic surgery; d) final result after bimaxillary surgery

diastema is achieved. During the postoperative latency period of approx. 7 days, the patient is instructed how to handle the distractor. The maxillary expansion protocol involves expanding the screw by quarter-turn twice daily ( $0.4 \mathrm{~mm}$ a day), until the desired arch widths and interarch relationships are achieved (Fig. 9-10). 


\section{Discussion}

Maxillary constriction is clinically manifested as a narrow palate and a high palatal vault, a complete unilateral or bilateral crossbite and dark buccal corridors. Structural maxillary abnormality may also be associated with such functional impairments as food chewing disorder, nose breathing problems and a development of a mouth breathing habit.

According to Laptok [32], a typical developmental maxillary constriction can be diagnosed based on the presence of a high palatal vault, a narrow palate and at least two of the following symptoms:

1. Progressively worsening nasal patency up to a complete stenosis, where nose breathing becomes impossible;

2. Nasal floor elevation;

3. Open-mouth breathing;

4. A complete bilateral crossbite with the high palatal vault;

5. Enlarged nasal turbinates, which impair nasal patency.

Obviously, not all clinical features of transverse maxillary deficiency are present in each case. Some of these traits may be more pronounced, whereas others are significantly less severe.

Transverse maxillary deficiency poses a significant challenge to orthodontic treatment. Orthodontic approaches aiming at buccal inclination are often insufficient as a stand-alone treatment to achieve stable occlusal relationships, which determine permanent therapeutic success. Most authors agree that the orthodontic treatments exert their dentoalveolar effect, whereas surgical approaches result in the actual increase of the maxillary bone base $[2,8,9,14,17,18,27,28]$.

Midpalatal suture expansion by means of increasing the maxillary bone base and osteogenesis is an effective treatment of maxillary constriction.

All methods of SARPE/SARME and transpalatal distraction (TPD) are based on the theory of distraction osteogenesis (DO) as the main assumption for the increase of the maxillary bone base. In 1905, Codivilla was the first to describe the technique of lengthening long bones using distraction osteogenesis. The method was improved and popularized de novo by Gawril Ilizarov, the Russian orthopaedic surgeon, who in 1950 developed and applied biological assumptions of distraction osteogenesis to lengthen or reshape limb bones. His invention, currently known as Ilizarov apparatus, consists of several metal rings connected with rods and has been successfully used in orthopaedic treatment until now.

Distraction osteogenesis is a bone growth inducing technique, which involves a controlled, gradual stretching of the primary callus forming within the post-osteotomy bed. It is a four-stage process involving corticotomy (osteotomy), latency, distraction and retention. Within the first 5 days following corticotomy, the osteotomy slit is filled with a blood clot. Clinically, this period is equivalent to the postoperative latency. Next, at 5 to 7 days, the collagen fibres are formed, which grow into the osteotomy slit. The collagen fibres are parallel to the distraction vector. The proven distraction protocol involves expansion by 0.25 to $1.0 \mathrm{~mm}$ a day continued until the planned bone tissue increase is achieved. An active treatment is followed by the retention period essential for the newly formed osseous tissue to mature without the unwanted exposure to motion or weightbearing. The advantages of osteodistraction include osteogenesis and lengthening of the soft tissues by applying low force for some period of time [33].

Surgical maxillary expansion may be a source of potential problems. Hence, some patients find it difficult to accept and consent to. The associated costs may pose the biggest problem. Furthermore, surgical treatment is always related to the risk of intra- and postoperative complications as well as simple postoperative inconvenience. Palatal distraction itself carries a risk of such complications as assymetrical expansion, clinical attachment loss on incisors, tooth loss due to necrosis, root resorption, osteonecrosis and soft tissue injury. The postoperative inconvenience includes oedema, pain, abnormal sensation, difficulties swallowing, breathing and speaking. Treatment-related work absenteeism may also be a problem. The distraction involves forming a large, unaesthetic diastema, which takes time to close. Some patients may find it frustrating and difficult to cope with [34].

Both orthodontic and surgical maxillary expansion approaches can be effective and yield good outcomes. However, they should be used based on clear diagnostic standards. Surgery is intended for patients with confirmed skeletal maturity, where good outcomes of orthodontic treatment are relatively unlikely.

Orthodontic treatment is most effective at the developmental age and the best outcomes are achieved in individuals before the pubertal growth spurt. The choice of treatment approach depending on clinical indications determines the stability of outcomes and the overall therapeutic success. Many authors report the loss of a third of the anterior arch width in prepubertal children and adolescents treated with rapid palatal expansion (RPE).

The main indication of SARPE and TPD is a transverse maxillary deficiency of over $5 \mathrm{~mm}$ in 
an adult. The reported recurrence after SARME ranges between $4 \%$ and $50 \%$ [19]. It is difficult to determine the origin of such huge discrepancies in the literature. A theory has been proposed to explain the mechanism of recurrence after maxillary constriction treatment with RME and SARME. It was postulated that the mechanical strength of orthodontic appliances used for maxillary expansion is insufficient to overcome the forces generated by the facial muscles and soft tissue, whose aim is to restore the baseline anatomical relationships $[28,35,36]$. Nevertheless, the transpalatal distraction (TPD) used for treatment of maxillary constriction does not seem to support the above theory. Using a transpalatal distractor for maxillary expansion, Matteini and Mommaerts [36] did not observe recurring maxillary constriction and maxillary transverse dimension loss after treatment. Zahl and Gerlach [37] shared these observations and did not recommend overcorrection for transpalatal distraction, as they did not observe recurrence in their patients after TPD.
It also seems that in many cases the recurrence is caused primarily by the unstable occlusal relationships after the treatment has been completed $[17,38,39]$.

\section{Conclusions}

The choice of the appropriate treatment approach in transverse maxillary deficiency should be strictly based on patient's age, skills and expertise of the therapeutic team as well as patient's expectations.

Orthodontic treatment should be considered in patients at the developmental age, whereas surgery is intended for patients with confirmed skeletal maturity.

Midpalatal suture expansion with the increase of the maxillary bone base results in an improved occlusion and facial aesthetic appearance. Additionally, it affects the ENT aspects, including improved nose breathing in treated individuals.

Acknowledgment. The authors thank dr Liwia Minch for sharing Fig. 6.

\section{References}

[1] Ciambotti C., Nagan P., Durknee M., Kohli K., Kim H.: A comparsion of dental and dentoalveolar beetween rapid palatal expansion and nickiel-titanium palatal expansion appliances. Am. J. Orthod. Dent. Orthop. 2001, 119, $11-20$.

[2] Mommaerts M.Y.: Transpalatal distraction as a method of maxillary expansion. Br. J. Oral Maxillofacial. Surg. 1999, 37, 268-272.

[3] Wertz R.A.: Rapid expansion of the maxillary dental arch and nasal cavity by opening the mid-palatal suture. Angle Orthod. 1961, 31, 73-90.

[4] Hats A.J.: Palatal expansion: Just the beginning of dentofacial orthopedics. Am. J. Orthod. 1970, 57, $219-255$.

[5] Kamińska I.: Orthodontic effects of midpalatal suture separation. Ann. Acad. Med. Stettin. 2008, 54, 1, 94-105 [in Polish].

[6] Proffit W.R., Fields H.W.: Ortodoncja współczesna. Wyd. Czelej, Lublin 2000.

[7] Persson M., Thilander B.: Palatal suture closure in man from 15 to 35 years of age. Am. J. Orthod. 1977, 72, 42-45.

[8] Bishara S.E., Stale R.N.: Maxillary expansions: clinical implications. Am. J. Orthod. Dentofacial Orthop. 1987, 91, 3-14.

[9] Filhoa R.L., Ruellas A.C.: Long-term maxillary changes in patients with skeletal Class II malocclusion treated with slow and rapid palatal expansion. Am. J. Orthod. Dentofacial Orthop. 2008,134, 383-388.

[10] Mustafa A., Mustafa E., Kurzawski M.: Orthodontic method of maxillary expansion. Dental Forum. 2005, 33 , 2, 75-79 [in Polish].

[11] Kurzawski M., Biedziak B.: Construction and function of nickiel titanium palatal expande (NPE-2). Dent. Med. Probl. 2004, 41, 549-552 [in Polish].

[12] Erdnic A.E., UĐur T., Erbay E.: A comparison of different treatment techniques for posterior cross bite in mixed dentition. Am. J. Orthod. Dentofacial Orthop. 1999, 115, 569-575.

[13] Lagravere M.O., Carey J.P., Heo G., Toogood R.W., Major P.W.: Transverse, vertical, and anteroposterior changes from bone-anchored maxillary expansion vs traditional rapid maxillary expansion: randomized clinical trial. Am. J. Othod. Dentofacial Orthop. 2010, 137, 304-305.

[14] Weissheimer A., De Menezes L.M., Mezomo M., Dias D.M., De Lima E.M.S., Rizzatto S.M.D.: Immediate effects of rapid maxillary expansion with Haas-type and hyrax-type expanders: a randomized clinical trial. Am. J. Othod. Dentofacial Orthop. 2011, 140, 366-376.

[15] Garib D.G., Henriques J.F., Janson G., De freitas M.R., Fernandes A.Y.: Periodontal effects of rapid maxillary expansion with tooth-tissue-borne and tooth-tissue expanders: a computed tomography evaluation. Am. J. Orthod. Dentofacial Orthop. 2006, 129, 749-758.

[16] KarŁowska I.: Contemporary orthodontics. Wydawnictwo Lekarskie PZWL, Warszawa 2009, 269-270 [in Polish].

[17] Clark W.J.: Transforce lingual appliances for arch development. J. Clin. Orthodont. 2005, 39, 137-142. 
[18] Talapaneni A.K., Kumar K.P., Kommi P.B., Nuvvula S.: Interceptive orthopedics for the correction of maxillary transverse and sagittal deficiency in the early mixed dentition period. Contemp. Clin. Dent. 2011, 11, 331-336.

[19] Chrcanovic B.R., Custódio A.L.: Orthodontic or surgically rapid maxillary expansion. Oral Maxillofac. Surg. 2009, 13, 123-137.

[20] Suri L., Taneja P.: Surgically assisted rapid palatal expansion: a literature review. Am. J. Orthod. Dentofacial Orthop. 2008, 133, 290-302.

[21] Baratieri C., Alves M., de Souza M.M.G., De Souza Araujo M.T., Maia L.C.: Does rapid maxillary expansion have long-term effects on airway dimensions and breathing? Am. J. Othod. Dentofacial Orthop. 2011, 140, 146-156.

[22] Boysen B., la Cour K., Athanasiou A.E., Gjessing P.E.: Three-dimensional evaluation of dentoskeletal changes after posteriori cross-bite correction by quad-helix or removable appliances. Br. J. Orthod. 1992, 19, 97-107.

[23] Vizzotto M.B., de Araujo F.B., Da Silveira H.S., Boza A.A., Closs L.Q.: The quad-helix appliance in the dentition-orthodontic and orthopedic measurement. J. Clin. Pediatr. Dent. 2007, 32, 165-170.

[24] Frank S.W., ENGEL G.A.: The effects of maxillary quad-helix appliance expansion on cephalometric measurement in growing orthodontic patients. Am. J. Orthod. 1982, 81, 378-389.

[25] Sandikcioglu M., Hazar S.: Skeletal and dental changes after maxillary expansion in the mixed dentition. Am. J. Orthod. Dentofacial Orthop. 1997, 111, 321-327.

[26] VÉGH A., Dénes Z., Razouk G., Dénes J.: The development and action-mechanism of the quadhelix in orthodontic treatment. Fogorv Sz. 1999, 92, 143-150.

[27] MCNally M.R., Spary D.J.: A randomized controlled trial comparing the quadhelix and the expansion arch for the correction of crossbite. J. Orthod. 2005, 32, 29-35.

[28] Huynh T., Kennedy D.B., Joondeph D.R., Bollen A.M.: Treatment response and stability of slow maxillary expansion using Haas, hyrax, and quad-helix appliances - a retrospective study. Am. J. Orthod. Dentofacial Orthop. $2009,136,331-339$.

[29] Handelman C.: Palatal expansion in adults: the nonsurgical approach. Am. J. Orthod. Dentofacial Orthop. 2011, $140,4-11$.

[30] IsERI H., Ozsoy S.: Semirapid maxillary expansion - a study of long-term transverse effects in older adolescents and adults. Angle Orthod. 2004, 74, 71-78.

[31] Kartalian A., Gohl E., Adamian M., Enciso R.: Cone-beam computerized tomography evaluation of the maxillary dentoskeletal complex after rapid palatal expansion. Am. J. Orthod. Dentofacial Orthop. 2010, 138, 486-492.

[32] Laptook T.: Conductive hearing loss and rapid maxillary expansion. Am. J. Orthod. 1981, 80, 325-331.

[33] Kulewicz M.: Dystraction osteoganesis in orthognathic surgery. Acta Cinica 2001, 2, 117-128.

[34] Northway W.: Palatal expansion in adults: the surgical approach. Am. J. Orthod. Dentofacial Orthop. 2011, 140, 463-469.

[35] Schwarz G.M., Thrash W.J., Byrd D.L., Jacobs J.D.: Tomographic assessment of nasal septal changes following surgical - orthodontic rapid maxillary expansion. Am. J. Orthod. Dentofacial Orthop.1985, 87, 39-45.

[36] Matteini C., Mommaerts M.Y.: Posterior transpalatal distraction with pterygoid disjunction: a short-term model study. Am. J. Orthod. Dentofacial Orthop. 2001, 120, 498-502.

[37] Zahl C., Gerlach K.L.: Palatinaldistraktor: ein innovativer Ansatz fur die Gaumennahterweiterung. Mund. Kiefer Gesichts Chir. 2002, 24, 446-449.

[38] Sтrömberg C., Holm J.: Surgically assisted rapid maxillary expansion in adults. A retrospective long-term followup study. J. Craniomaxillofac. Surg. 1995, 23, 222-227.

[39] Koudstaal M.J., Wolvius E.B., Schulten A.J., Hop W.C., Van der WaAl K.G.: Stability, tipping and relapse of bone-borne versus tooth-borne surgically assisted rapid maxillary expansion; a prospective randomized patient trial. Int. J. Oral Maxillofac. Surg. 2001, 38, 308-315.

\section{Address for correspondence:}

Rafał Nowak

Department of Maxillofacial Surgery

Wroclaw Medical University

50-556 Wrocław

Borowska 213

Tel.: 0717343695

E-mail: rafal.nowak@chirurgiatwarzy.pl

Conflict of Interest: None declared

Received: 06.07.2015

Revised: 02.08.2015

Accepted: 11.09.2015 